Hardy-Ramanujan Journal

Vol. 33 (2010) pp. 59-70

\title{
Some diophantine problems concerning equal sums of integers and their cubes
}

\author{
Ajai Choudhry \\ Dean, Foreign Service Institute, \\ Old J. N. U. Campus, Baba Gang Nath Marg, \\ New Delhi-110067, INDIA \\ E-mail: ajaic203@yahoo.com
}

\begin{abstract}
This paper gives a complete four-parameter solution of the simultaneous diophantine equations $x+y+z=u+v+w, x^{3}+y^{3}+z^{3}=$ $u^{3}+v^{3}+w^{3}$, in terms of quadratic polynomials in which each parameter occurs only in the first degree. This solution is much simpler than the complete solutions of these equations published earlier. This simple solution is used to obtain solutions of several related diophantine problems. For instance, the paper gives a parametric solution of the arbitrarily long simultaneous diophantine chains of the type $x_{1}^{k}+y_{1}^{k}+z_{1}^{k}=x_{2}^{k}+y_{2}^{k}+z_{2}^{k}=\ldots=x_{n}^{k}+y_{n}^{k}+z_{n}^{k}=\ldots, \quad k=1,3$. Further, the complete ideal symmetric solution of the Tarry-Escott problem of degree 4 is obtained, and it is also shown that any arbitrarily given integer can be expressed as the sum of four distinct nonzero integers such that the sum of the cubes of these four integers is equal to the cube of the given integer.
\end{abstract}

Keywords: equal sums of powers; equal sums of cubes;

Tarry-Escott problem; diophantine chains.

Mathematics Subject Classification: 11D25

\section{Introduction}

This paper is concerned with the simultaneous diophantine equations,

$$
\begin{aligned}
x+y+z & =u+v+w, \\
x^{3}+y^{3}+z^{3} & =u^{3}+v^{3}+w^{3},
\end{aligned}
$$

and various related diophantine problems. Gerardin gave partial solutions of these simultaneous equations in 1915-16 (as quoted by Dickson [8, pp. 565,713 ] and additional partial solutions were given by Bremner [1]. Subsequently, complete solutions (apart from a multiplicative factor) were given in terms of cubic polynomials in four variables by Bremner and Brudno [2], 
as well as by Labarthe [9]. A complete solution in terms of eight variables was given by Choudhry [4, p. 299-301].

We note that since each of the equations (1) and (2) is homogeneous, if $x, y, z, u, v, w$, is a solution in rational numbers of these simultaneous equations, so is $\rho x, \rho y, \rho z, \rho u, \rho v, \rho w$, where $\rho$ is any nonzero rational number, and all such proportional solutions will be considered equivalent. It follows that any solution of the simultaneous equations (1) and (2) in rational numbers, yields on appropriate scaling, a solution in integers.

In this paper we give, in Section 2, a new complete solution of the simultaneous diophantine equations (1) and (2) in terms of quadratic polynomials in four parameters such that each parameter appears in the solution only in the first degree. This solution is thus much simpler than all the complete solutions published earlier and is of particular interest since it yields solutions to various other related diophantine problems, and also because the diophantine system given by (1) and (2) has applications in mathematical physics $[1,2,3,9]$. As an immediate consequence of this solution, we obtain the complete solution of the diophantine system

$$
x^{k}+y^{k}=u^{k}+v^{k}+w^{k}, \quad k=1,3,
$$

for which only a partial solution had been given earlier by Gerardin (as quoted in $[8$, p. 708]).

The simple solution of Section 2 is used in Section 3 to obtain two parametric solutions of the equations (1) and (2) which are in turn used in Section 4 to obtain arbitrarily long simultaneous diophantine chains of the type

$$
x_{1}^{k}+y_{1}^{k}+z_{1}^{k}=x_{2}^{k}+y_{2}^{k}+z_{2}^{k}=\ldots=x_{n}^{k}+y_{n}^{k}+z_{n}^{k}=\ldots, \quad k=1,3 .
$$

While a method of generating numerical chains of this type was earlier given by Choudhry [5], in this paper a parametric solution of these chains is given. We further show how to generate arbitrarily long simultaneous diophantine chains of the type

$$
\begin{aligned}
& x_{1}+y_{1}+z_{1}=x_{2}+y_{2}+z_{2}=\ldots=x_{n}+y_{n}+z_{n}=\ldots, \\
& x_{1}^{3}+y_{1}^{3}+z_{1}^{3}=x_{2}^{3}+y_{2}^{3}+z_{2}^{3}=\ldots=x_{n}^{3}+y_{n}^{3}+z_{n}^{3}=\ldots, \\
& x_{1} y_{1} z_{1}=x_{2} y_{2} z_{2}=\ldots=x_{n} y_{n} z_{n}=\ldots
\end{aligned}
$$

In Section 5 we use the results of Section 2 to obtain the complete ideal symmetric solution of the Tarry-Escott problem of degree 4. This solution is much simpler than the complete solution of this problem given earlier in [6]. We also show that any arbitrarily given integer can be expressed as the sum of four distinct nonzero integers such that the sum of the cubes of these four integers is equal to the cube of the given integer. Finally, we obtain a parametric solution of the simultaneous diophantine equations

$$
\begin{aligned}
X+Y+Z & =3, \\
X^{3}+Y^{3}+Z^{3} & =3 .
\end{aligned}
$$




\section{The complete solution of the simultaneous dio- phantine equations (1) and (2)}

It is easily seen that all trivial solutions of the equations (1) and (2) are given by $x, y, z$ being a permutation of $u, v, w$, or by $x, y, z$ being taken as $\{f,-f, h\}$ in some order and $u, v, w$ being taken as $\{g,-g, h\}$ in some order where $f, g, h$ are arbitrary. We now prove a theorem that gives all nontrivial solutions of these equations.

Theorem 1: All nontrivial solutions of the simultaneous diophantine equations (1) and (2), apart from a multiplicative factor, are given by

$$
\begin{aligned}
x & =p q-p r+q r+(-p+q+r) s \\
y & =-p q+p r+q r+(p-q+r) s \\
z & =p q+p r-q r+(p+q-r) s \\
u & =p q+p r-q r+(p-q+r) s \\
v & =p q-p r+q r+(p+q-r) s \\
w & =-p q+p r+q r+(-p+q+r) s
\end{aligned}
$$

where $p, q, r$ and $s$ are arbitrary integer parameters.

Proof: To solve the above diophantine system, we make the invertible linear transformation given by

$$
\begin{aligned}
& x=-X+Y+Z, \quad u=-U+V+W, \\
& y=X-Y+Z, \quad v=U-V+W \\
& z=X+Y-Z, \quad w=U+V-W,
\end{aligned}
$$

and

$$
\begin{aligned}
& X=(y+z) / 2, \quad U=(v+w) / 2, \\
& Y=(z+x) / 2, \quad V=(w+u) / 2, \\
& Z=(x+y) / 2, \quad W=(u+v) / 2,
\end{aligned}
$$

when equations (1) and (2) are transformed to the following two equations respectively:

$$
\begin{aligned}
X+Y+Z & =U+V+W \\
(X+Y+Z)^{3}-24 X Y Z & =(U+V+W)^{3}-24 U V W .
\end{aligned}
$$

In view of (10), equation (11) reduces to

$$
X Y Z=U V W .
$$

To solve the simultaneous equations (10) and (12), we write

$$
\begin{aligned}
& X=p a, Y=q b, Z=r c, \\
& U=q a, V=r b, W=p c,
\end{aligned}
$$


where $p, q, r, a, b, c$, are arbitrary parameters. With these values of $X, Y, Z$, $U, V, W$, equation (12) is identically satisfied while equation (10) becomes,

$$
(p-q) a+(q-r) b+(r-p) c=0 .
$$

It is readily seen that a solution of (14) may be written as

$$
a=r+s, \quad b=p+s, \quad c=q+s,
$$

where $s$ is an arbitrary parameter. Substituting these values of $a, b, c$ in (13), we obtain the values of $X, Y, Z, U, V, W$ which, on being substituted in (8) yield the solution (7) of the simultaneous equations (1) and (2).

It is clear that integer values of the parameters $p, q, r$ and $s$ generate integer solutions of the diophantine system given by (1) and (2). We shall now show that conversely, any nontrivial integer solution of these equations is generated by suitably chosen integer values of the parameters $p, q, r$ and $s$.

Let $(x, y, z, u, v, w)=(\alpha, \beta, \gamma, \xi, \eta, \zeta)$ be any given nontrivial integer solution of the diophantine system given by the equations (1) and (2) so that,

$$
\begin{aligned}
\alpha+\beta+\gamma & =\xi+\eta+\zeta, \\
\alpha^{3}+\beta^{3}+\gamma^{3} & =\xi^{3}+\eta^{3}+\zeta^{3} .
\end{aligned}
$$

It follows that

$$
(\alpha+\beta+\gamma)^{3}-\left(\alpha^{3}+\beta^{3}+\gamma^{3}\right)=(\xi+\eta+\zeta)^{3}-\left(\xi^{3}+\eta^{3}+\zeta^{3}\right),
$$

which, on simplification, gives the relation,

$$
(\alpha+\beta)(\beta+\gamma)(\gamma+\alpha)=(\xi+\eta)(\eta+\zeta)(\zeta+\xi) .
$$

We will show that the parametric solution (7) generates the given solution $(\alpha, \beta, \gamma, \xi, \eta, \zeta)$ if we choose the following integer values of the parameters $p, q, r, s$ :

$$
\begin{aligned}
& p=(\alpha+\gamma)(\beta+\gamma)(\beta-\xi) \\
& q=(\alpha+\gamma)(\beta-\xi)(\eta+\zeta) \\
& r=(\beta-\xi)(\xi+\zeta)(\eta+\zeta) \\
& s=(\alpha-\eta)(\alpha+\gamma)(\eta+\zeta)
\end{aligned}
$$

With these values of the parameters, and with $x, y, z, u, v, w$ defined by (7), the following relations are readily verified:

$$
\begin{aligned}
& (\eta+\zeta) y+(\eta+\zeta) z-(\beta+\gamma) v-(\beta+\gamma) w=0 \\
& (\xi+\zeta) x+(\xi+\zeta) z-(\alpha+\gamma) u-(\alpha+\gamma) w=0
\end{aligned}
$$


Further, we find that

$$
\begin{aligned}
\frac{x+y}{u+v} & =\frac{(\xi+\zeta)(\eta+\zeta)}{(\beta+\gamma)(\alpha+\gamma)} \\
& =\frac{\alpha+\beta}{\xi+\eta}, \quad \text { in view of }(19)
\end{aligned}
$$

which gives the relation,

$$
(\xi+\eta) x+(\xi+\eta) y+(-\alpha-\beta) u+(-\alpha-\beta) v .
$$

Similarly we find that

$$
\frac{x+z}{v+w}=\frac{\alpha+\gamma}{\alpha+\beta+\gamma-\xi}=\frac{\alpha+\gamma}{\eta+\zeta}, \quad \text { in view of (16), }
$$

which gives the relation,

$$
(\eta+\zeta) x+(\eta+\zeta) z+(-\alpha-\gamma) v+(-\alpha-\gamma) w .
$$

We also note that $x, y, z, u, v, w$ defined by (7) identically satisfy the relation (1). Thus, with $p, q, r, s$ defined by (20), the solution $x, y, z, u, v, w$ given by (7) satisfies the five linear equations (1), (21), (22), (24) and (26). Applying Cramer's rule to solve these five equations, and repeatedly using the relation (16) for simplification, we get

$$
\frac{x}{k \alpha}=\frac{y}{k \beta}=\frac{z}{k \gamma}=\frac{u}{k \xi}=\frac{v}{k \eta}=\frac{w}{k \zeta},
$$

where

$$
k=-2(\alpha+\gamma)(\eta+\zeta)(\gamma-\zeta) .
$$

Since $(\alpha, \beta, \gamma, \xi, \eta, \zeta)$ is a nontrivial solution of the equations (1) and (2), we must have $k \neq 0$, and it now follows from (27) that

$$
x: y: z: u: v: w=\alpha: \beta: \gamma: \xi: \eta: \zeta .
$$

This shows that when we choose $p, q, r$ and $s$ as given by (20), the solution generated by (7) is equivalent to the given solution. Thus all nontrivial solutions of the simultaneous equations (1) and (2) are generated by taking suitable integer values of the parameters $p, q, r, s$ in the solution (7). This proves the theorem.

We note that (7) also generates some, but not all, trivial solutions of (1) and (2). For instance, it follows from (20) and the above computations that (7) generates the trivial solution

$$
x=\alpha, \quad y=\beta, \quad z=\gamma, \quad u=\alpha, \quad v=\gamma, \quad z=\beta,
$$


where $\alpha, \beta, \gamma$ are arbitrary, if we choose the following values of the parameters $p, q, r$ and $s$ :

$$
\begin{aligned}
& p=(\alpha-\beta)(\alpha+\gamma)(\beta+\gamma) \\
& q=(\alpha-\beta)(\alpha+\gamma)(\beta+\gamma), \\
& r=(\alpha-\beta)(\alpha+\beta)(\beta+\gamma) \\
& s=-(\alpha-\gamma)(\alpha+\gamma)(\beta+\gamma)
\end{aligned}
$$

This is also readily verified by direct computation.

We also note that if $p, q, r$ are chosen to satisfy the triangle inequality, the coefficients of $s$ in the values of $x, y, z, u, v, w$ given by (7) are all positive, and hence we can readily choose values of $s$ such that (7) gives a solution of equations (1) and (2) in positive integers. As a numerical example, when $p=2, q=3, r=4, s=1$, we get the following solution:

$$
3^{k}+15^{k}+17^{k}=5^{k}+11^{k}+19^{k}, \quad k=1,3 .
$$

Corollary 2: All nontrivial solutions of the simultaneous diophantine equations

$$
x^{k}+y^{k}=u^{k}+v^{k}+w^{k}, \quad k=1,3,
$$

are given by

$$
\begin{aligned}
x & =\left(p^{2}-2 p r+q r\right) q, \\
y & =-\left(p q-2 q r+r^{2}\right) p, \\
u & =(q-r)(p q+p r-q r), \\
v & =(-p-q+r)(p-q) r, \\
w & =(p-r)\left(p r-q^{2}\right),
\end{aligned}
$$

where $p, q, r$ are arbitrary parameters.

Proof: Since the theorem gives all nontrivial solutions of the simultaneous equations (1) and (2), and this solution is linear in each of the parameters, it readily yields all nontrivial solutions of the equations (1) and (2) with $z=0$. By choosing the parameter $s$ such that $z=0$, we get all nontrivial solutions of the diophantine system (32) stated above. We further note that by imposing the condition obtained by equating any one of $x, y, u, v, w$ to zero, and choosing $s$ accordingly, we obtain solutions of (32) that are effectively the same as the solution (33). This completes the proof.

\section{Two parametric solutions}

We now use the complete solution given in Theorem 1 to derive two parametric solutions of the simultaneous equations (1) and (2). These parametric solutions will be used in the subsequent sections to obtain several new relations concerning equal sums of integers and their cubes. 
Theorem 2: Two parametric solutions of the simultaneous equations (1) and (2) are given by

$$
x=\alpha, \quad y=\beta, \quad z=\gamma, \quad u=\alpha_{1}, \quad v=\beta_{1}, \quad w=\gamma_{1},
$$

where

$$
\begin{aligned}
& \alpha_{1}=\frac{\left(\alpha^{3}-\alpha \beta^{2}-\alpha \beta \gamma-\alpha \gamma^{2}+\beta^{3}+\gamma^{3}\right)}{(\alpha-\beta)(\alpha-\gamma)}, \\
& \beta_{1}=\frac{\left(\alpha^{3}-\alpha^{2} \gamma-\alpha \beta \gamma+\beta^{3}-\beta^{2} \gamma+\gamma^{3}\right)}{(\alpha-\gamma)(\beta-\gamma)}, \\
& \gamma_{1}=-\frac{\left(\alpha^{3}-\alpha^{2} \beta-\alpha \beta \gamma+\beta^{3}-\beta \gamma^{2}+\gamma^{3}\right)}{(\alpha-\beta)(\beta-\gamma)},
\end{aligned}
$$

with $\alpha, \beta, \gamma$ being arbitrary parameters, and by

$$
\begin{aligned}
x=\mu & \left(8 \mu^{2}+27 \mu+27\right), \\
y=- & (2 \mu+3)\left(5 \mu^{2}+15 \mu+18\right), \\
z= & (\mu+3)\left(8 \mu^{2}+21 \mu+18\right), \\
u=\mu\left\{2 \mu^{4}+\left(12 \mu^{4}+108 \mu^{3}+351 \mu^{2}+486 \mu+243\right) \nu^{2}\right. & \\
& \left.+\left(6 \mu^{4}+27 \mu^{3}+27 \mu^{2}\right) \nu\right\}\left\{\left(2 \mu^{2}+9 \mu+9\right) \nu^{2}+\mu^{2} \nu\right\}^{-1}, \\
v=( & +3)(2 \mu+3)\left\{3 \mu^{4}+\left(8 \mu^{4}+72 \mu^{3}+234 \mu^{2}+324 \mu+162\right) \nu^{2}\right. \\
& \left.+\left(6 \mu^{4}+27 \mu^{3}+27 \mu^{2}\right) \nu\right\}\left\{\mu^{3}+\left(2 \mu^{2}+9 \mu+9\right) \mu \nu\right\}^{-1}, \\
w=- & \left\{2 \mu^{4}+\left(8 \mu^{4}+72 \mu^{3}+234 \mu^{2}+324 \mu+162\right) \nu^{2}\right. \\
& \left.+\left(2 \mu^{4}+9 \mu^{3}+9 \mu^{2}\right) \nu\right\}(\mu \nu)^{-1},
\end{aligned}
$$

where $\mu$ and $\nu$ are arbitrary parameters.

Proof: We will now use the complete solution (7) of equations (1) and (2) to derive another solution of these equations in which $x, y, z$ are proportionate to $\alpha, \beta, \gamma$, that is, the solution satisfies the simultaneous equations

$$
\begin{gathered}
\beta x=\alpha y, \\
\gamma x=\alpha z,
\end{gathered}
$$

where $\alpha, \beta, \gamma$ are arbitrary. Substituting the values of $x, y, z$ given by (7) in (37) and (38), we get

$$
\begin{aligned}
& \beta\{p q-p r+q r+(-p+q+r) s\}=\alpha\{-p q+p r+q r+(p-q+r) s\}, \\
& \gamma\{p q-p r+q r+(-p+q+r) s\}=\alpha\{p q+p r-q r+(p+q-r) s\} .
\end{aligned}
$$

To solve these simultaneous equations, we first observe that the trivial solution (30), which satisfies the equations (37) and (38), is generated by the values of $p, q, r, s$ given by (31). Therefore, these values of $p, q, r, s$ satisfy the simultaneous equations (39) and (40) and we will use this solution to obtain another solution of these equations. To obtain a rational solution of (39) and (40), we must choose $p, q, r$ such that the following condition, obtained by eliminating $s$ from (39) and (40) is satisfied:

$$
(\alpha+\beta) p^{2} q-2(\alpha+\beta+\gamma) p q r+(\alpha+\gamma) p r^{2}+(\beta+\gamma) q^{2} r=0 .
$$


Treating $\alpha, \beta, \gamma$ as arbitrary constants, equation (41) is a cubic equation in $p, q, r$, which represents a curve of genus 1 in the projective plane. Using the known rational point on this curve given by (31), we readily find another rational point on the curve (41) which is as follows:

$$
\begin{aligned}
& p=(\alpha-\gamma)^{2}(\alpha+\gamma)(\beta-\gamma)(\beta+\gamma) \\
& q=(\alpha+\beta)(\alpha-\beta)^{2}(-\alpha+\gamma)(\alpha+\gamma) \\
& r=(\alpha+\beta)(\alpha-\beta)(\beta+\gamma)(\beta-\gamma)^{2}
\end{aligned}
$$

With these values of $p, q, r$, equation (41) is satisfied and both equations (39) and (40) are satisfied by the same rational value of $s$. We have thus solved the simultaneous equations (39) and (40) for $p, q, r, s$ with $\alpha, \beta, \gamma$ being arbitrary. Substituting these values of $p, q, r, s$ in (7), we get a solution which, on appropriate scaling, yields the solution given by (34) and (35) as stated in the theorem.

While equation (41) represents a curve of genus 1 for arbitrary values of $\alpha, \beta, \gamma$, it is possible to choose values of $\alpha, \beta, \gamma$, such that the curve represented by (41) has a singularity and thus reduces to a curve of genus 0 . This happens when we choose

$$
\begin{aligned}
\alpha & =\left(8 \mu^{2}+27 \mu+27\right) \mu \\
\beta & =-(2 \mu+3)\left(5 \mu^{2}+15 \mu+18\right) \\
\gamma & =(\mu+3)\left(8 \mu^{2}+21 \mu+18\right)
\end{aligned}
$$

where $\mu$ is arbitrary, and with these values, the cubic curve (41) has a singularity given by

$$
p=-\mu^{2}(2 \mu+3), \quad q=(\mu+3)(2 \mu+3)^{2}, \quad r=\mu(\mu+3)^{2},
$$

and we readily find another point on the curve (41) which is given by

$$
\begin{aligned}
& p=\left(2 \mu^{2} \nu+\mu^{2}+9 \mu \nu+9 \nu\right)^{2} \nu, \\
& q=\left(2 \mu^{2} \nu+\mu^{2}+9 \mu \nu+9 \nu\right) \mu^{2}, \\
& r=-(\mu+3)^{3} \mu \nu^{2},
\end{aligned}
$$

where $\nu$ is an arbitrary parameter. Proceeding as before, we obtain the parametric solution (36) stated in the theorem.

The significance of the first parametric solution given in the theorem is that, in general, given any three arbitrary rational numbers $\alpha, \beta$, $\gamma$, we can find another set of three rational numbers $\alpha_{1}, \beta_{1}, \gamma_{1}$, with the same sum and the same sum of cubes. A more complicated solution of this type is given in [5]. We note that if we choose $\alpha, \beta, \gamma$ such that the sum of any two of these numbers is 0 , the formulae (35) yield a trivial solution. Similarly, we note that when the initial three rational numbers $\alpha, \beta, \gamma$, are taken as

$$
\lambda\left(\mu^{3}-\mu^{2}+1\right), \quad \lambda\left(\mu^{3}-3 \mu^{2}+2 \mu-1\right), \quad \lambda\left(-\mu^{3}+\mu^{2}-2 \mu+1\right),
$$


in some order, $\lambda, \mu$ being arbitrary parameters, then the formulae (35) yield the same set of numbers $\alpha, \beta, \gamma$, instead of giving a nontrivial solution of the problem.

The second solution given in the theorem is interesting because $x, y, z$ are given in terms of a single parameter while $u, v, w$ are given in terms of two parameters. We use this fact effectively in the next Section.

\section{Diophantine chains}

In this section we obtain arbitrarily long simultaneous diophantine chains involving equal sums of integers and their cubes.

Theorem 3: A parametric solution in rational numbers of the arbitrarily long simultaneous diophantine chains

$$
x_{1}^{k}+y_{1}^{k}+z_{1}^{k}=x_{2}^{k}+y_{2}^{k}+z_{2}^{k}=\ldots=x_{n}^{k}+y_{n}^{k}+z_{n}^{k}=\ldots, \quad k=1,3,
$$

is given by

$$
\begin{aligned}
x_{i}=\mu & \left\{2 \mu^{4}+\left(12 \mu^{4}+108 \mu^{3}+351 \mu^{2}+486 \mu+243\right) \nu_{i}^{2}\right. \\
& \left.+\left(6 \mu^{4}+27 \mu^{3}+27 \mu^{2}\right) \nu_{i}\right\}\left\{\left(2 \mu^{2}+9 \mu+9\right) \nu_{i}^{2}+\mu^{2} \nu_{i}\right\}^{-1}, \\
y_{i}=( & \mu+3)(2 \mu+3)\left\{3 \mu^{4}+\left(8 \mu^{4}+72 \mu^{3}+234 \mu^{2}+324 \mu+162\right) \nu_{i}^{2}\right. \\
& \left.+\left(6 \mu^{4}+27 \mu^{3}+27 \mu^{2}\right) \nu_{i}\right\}\left\{\mu^{3}+\left(2 \mu^{2}+9 \mu+9\right) \mu \nu_{i}\right\}^{-1}, \\
z_{i}=- & \left\{2 \mu^{4}+\left(8 \mu^{4}+72 \mu^{3}+234 \mu^{2}+324 \mu+162\right) \nu_{i}^{2}\right. \\
& \left.+\left(2 \mu^{4}+9 \mu^{3}+9 \mu^{2}\right) \nu_{i}\right\}\left(\mu \nu_{i}\right)^{-1}
\end{aligned}
$$

where $u$ and $v_{i}, i=1,2, \ldots, n, \ldots$, are arbitrary rational parameters.

Proof: The parametric solution (48) of the simultaneous diophantine chains (47) follows immediately from the solution (36) given in Theorem 2.

As a numerical example, taking $\mu=-1$, and giving to $\nu_{i}$ the values $1, \pm 2, \pm 3$, we get, on appropriate scaling, the following simultaneous diophantine chains:

$$
\begin{aligned}
(-840)^{k}+(-840)^{k}+1050^{k} & =(-1190)^{k}+(-700)^{k}+1260^{k} \\
& =(-1974)^{k}+(-651)^{k}+1995^{k} \\
& =(-2380)^{k}+(-644)^{k}+2394^{k} \\
& =(-2790)^{k}+(-640)^{k}+2800^{k}, \quad k=1,3 .
\end{aligned}
$$

We now give an alternative procedure of generating diophantine chains of the type (47). We have already observed that, with certain exceptions, given three arbitrary distinct rational numbers $\alpha, \beta, \gamma$, we can use the solution (34) to obtain rational numbers $\alpha_{1}, \beta_{1}, \gamma_{1}$, such that the relations

$$
\alpha^{k}+\beta^{k}+\gamma^{k}=\alpha_{1}^{k}+\beta_{1}^{k}+\gamma_{1}^{k}, \quad k=1,3,
$$


are satisfied. We can then repeat this process taking $\alpha_{1}, \beta_{1}, \gamma_{1}$, as the initial numbers in (34) and thus obtain rational numbers $\alpha_{2}, \beta_{2}, \gamma_{2}$, such that the relations

$$
\alpha_{1}^{k}+\beta_{1}^{k}+\gamma_{1}^{k}=\alpha_{2}^{k}+\beta_{2}^{k}+\gamma_{2}^{k}, \quad k=1,3,
$$

are satisfied. We can continue this process indefinitely and thus obtain a solution of the simultaneous diophantine chains (47). It can be proved, following the lines of a similar proof given in [5, pp. 136-137], that by a suitable choice of rational numbers $\alpha_{1}, \beta_{1}, \gamma_{1}$, we can obtain an arbitrarily long chain of the type (47).

We also note that in view of the identity,

$$
\alpha^{3}+\beta^{3}+\gamma^{3}-3 \alpha \beta \gamma=(\alpha+\beta+\gamma)\left(\alpha^{2}+\beta^{2}+\gamma^{2}-\alpha \beta-\beta \gamma-\gamma \alpha\right),
$$

if we choose the initial rational numbers $\alpha, \beta, \gamma$ such that $\alpha+\beta+\gamma=0$, then the above process will give arbitrarily long simultaneous diophantine chains of type (5).

As a numerical example, starting with the three rational numbers as $1,2,-3$, we get a chain which, on appropriate scaling, leads to the following simultaneous diophantine chains in integers:

$$
\begin{aligned}
& (-242606760)^{k}+80868920^{k}+161737840^{k} \\
& \quad=(-517561088)^{k}+12130338^{k}+505430750^{k} \\
& \quad=(-281011375)^{k}+48582831^{k}+232428544^{k}, \quad k=1,3,
\end{aligned}
$$

and

$$
\begin{aligned}
& (-242606760) \times 80868920 \times 161737840 \\
& \quad=(-517561088) \times 12130338 \times 505430750 \\
& =(-281011375) \times 48582831 \times 232428544 .
\end{aligned}
$$

Such diophantine chains have, in effect, been given earlier in [7, pp. 141-143] where the result is obtained and presented differently.

\section{Some additional results}

In this section we derive some new results from the solutions obtained in the previous sections.

5.1 Theorem 4: All nontrivial ideal symmetric solutions of the TarryEscott problem of degree 4, that is, of the diophantine system

$$
x_{1}^{k}+x_{2}^{k}+x_{3}^{k}+x_{4}^{k}+x_{5}^{k}=y_{1}^{k}+y_{2}^{k}+y_{3}^{k}+y_{4}^{k}+y_{5}^{k}, \quad k=1,2,3,4,
$$

are given by 


$$
\begin{aligned}
x_{1} & =\left(p^{2}-2 p r+q r\right) q, \\
x_{2} & =-\left(p q-2 q r+r^{2}\right) p, \\
x_{3} & =-(q-r)(p q+p r-q r), \\
x_{4} & =(p+q-r)(p-q) r, \\
x_{5} & =-(p-r)\left(p r-q^{2}\right), \\
y_{i} & =-x_{i}, \quad i=1,2,3,4,5,
\end{aligned}
$$

where $p, q, r$ are arbitrary parameters.

Proof: All symmetric solutions of the diophantine system (52) are obtained by writing $y_{i}=-x_{i}, \quad i=1,2, \ldots, 5$ when the problem reduces to solving the simultaneous equations

$$
x_{1}^{k}+x_{2}^{k}+x_{3}^{k}+x_{4}^{k}+x_{5}^{k}=0, \quad k=1,3 .
$$

We note that the simultaneous equations (32), on suitably transposing and renaming the variables, become exactly identical to the equations (54), and accordingly the solution (33) of (32) immediately yields all nontrivial solutions of (54). We thus obtain all nontrivial ideal symmetric solutions of the diophantine system (52) as stated in the theorem. This completes the proof.

5.2 Theorem 5: Any arbitrarily given integer can be expressed as the sum of four distinct nonzero integers such that the sum of the cubes of these four integers is equal to the cube of the given integer.

Proof: We substitute $p=N-1, q=-N, r=N$ in the solution (33) to get two identities which, on suitable transposition and renaming of variables, may be written as follows:

$$
\begin{aligned}
N & =N_{1}+N_{2}+N_{3}+N_{4}, \\
N^{3} & =N_{1}^{3}+N_{2}^{3}+N_{3}^{3}+N_{4}^{3},
\end{aligned}
$$

where

$$
\begin{array}{ll}
N_{1}=2 N^{3}, & N_{2}=\left(2 N^{2}-1\right) N, \\
N_{3}=-(2 N+1)(N-1) N, & N_{4}=-(N+1)(2 N-1) N .
\end{array}
$$

The above identities prove that all integers except $N \neq 0, \pm 1$, can be expressed as stated in the theorem. Further, the identities,

$$
\begin{aligned}
0 & =a^{k}+(-a)^{k}+b^{k}+(-b)^{k}, \quad k=1,3, \\
1 & =7+8+(-5)+(-9), \\
1^{3} & =7^{3}+8^{3}+(-5)^{3}+(-9)^{3}
\end{aligned}
$$

suffice to prove the theorem when $N=0, \pm 1$. This completes the proof.

5.3 Theorem 6: A parametric solution of the simultaneous diophantine equations

$$
\begin{aligned}
X+Y+Z & =3 \\
X^{3}+Y^{3}+Z^{3} & =3
\end{aligned}
$$


is given by

$$
\begin{aligned}
& X=u /\{\mu(\mu+3)(2 \mu+3)\}, \\
& Y=v /\{\mu(\mu+3)(2 \mu+3)\}, \\
& Z=w /\{\mu(\mu+3)(2 \mu+3)\},
\end{aligned}
$$

where $u, v, w$, are defined by (36) and $\mu, \nu$ are arbitrary parameters.

Proof: With $u, v, w$, defined by (36), it is readily verified that

$$
\begin{aligned}
u+v+w & =3 \mu(\mu+3)(2 \mu+3), \\
u^{3}+v^{3}+w^{3} & =3 \mu^{3}(\mu+3)^{3}(2 \mu+3)^{3} .
\end{aligned}
$$

It follows from these identities that when $X, Y, Z$, are defined by (58), then the simultaneous equations (57) are satisfied. This proves the theorem.

\section{References}

[1] A. Bremner, Diophantine equations and nontrivial Racah coefficients, J. Math. Phys. 27 (1986), pp. 1181-84.

[2] A. Bremner and S. Brudno, A complete determination of the zeros of weigh-1 6j coefficients, J. Math. Phys. 27 (1986), pp. 2613-2615.

[3] S. Brudno and J. D. Louck, Nontrivial zeros of weight $13 j$ and $6 j$ coefficients: Relation to diophantine equations of equal sums of like powers, J. Math. Phys. 26 (1985), pp. 2092-2095.

[4] A. Choudhry, Symmetric diophantine systems, Acta Arithmetica, 59 (1991), 291-307.

[5] A. Choudhry, Multigrade chains with odd exponents, Journal of Number Theory 42 (1992), 134-140.

[6] A. Choudhry, Ideal solutions of the Tarry-Escott problem of degree four and a related diophantine system, L'Enseignement Mathematique, 46 (2000), 313-323.

[7] A. Choudhry, Triads of cubes with equal sums and equal products, The Mathematics Student, 70 (2001), 137-143.

[8] L. E. Dickson, History of theory of numbers, Vol.2, Chelsea Publishing Company, New York, 1992, reprint.

[9] J. J. Labarthe, Parametrization of the linear zeros of $6 j$ coefficients, J. Math. Phys. 27 (1986), pp. 2964-65.

Received on January 16, 2010

Accepted on January 28, 2010 